\title{
ARTICLE \\ A pro-inflammatory CD8+ T-cell subset patrols the cervicovaginal tract
}

\author{
Laura Pattacini ${ }^{1}$, Amanda Woodward Davis ${ }^{1}$, Julie Czartoski ${ }^{1}$, Florian Mair ${ }^{1}$, Scott Presnell ${ }^{2}$, Sean M. Hughes ${ }^{3}$, Ollivier Hyrien ${ }^{1,4}$, \\ Gretchen M. Lentz ${ }^{3}$, Anna C. Kirby ${ }^{3}$, Michael F. Fialkow ${ }^{3}$, Florian Hladik ${ }^{3}$, Martin Prlic ${ }^{1}$ and Jennifer M. Lund ${ }^{1,5}$
}

The immune system of the cervicovaginal tract (CVT) must balance immunosurveillance and active immunity against pathogens with maintenance of tolerance to resident microbiota and to fetal and partner antigens for reproductive purposes. Thus, we predicted that CVT immunity is characterized by distinctive features compared to blood and other tissue compartments. Indeed, we found that CVT CD8 + T-cells had unique transcriptional profiles, particularly in their cytokine signature, compared to that reported for CD8+ T-cells in other tissue sites. Among these CVT CD8+ T-cells, we identified a CD69- CD103- subset that was characterized by reduced migration in response to tissue-exit signals and higher pro-inflammatory potential as compared to their blood counterpart. These inflammatory mucosal CD8 + T-cells (Tim) were increased in frequency in the CVT of individuals with chronic infection, pointing to a potential role in perpetuating inflammation. Our findings highlight the specialized nature of immunity within the CVT and identify Tim cells as potential therapeutic targets to tame tissue inflammation upon chronic infection.

Mucosal Immunology (2019) 12:1118-1129; https://doi.org/10.1038/s41385-019-0186-9

\section{INTRODUCTION}

Memory CD8+ T-cells play a critical role in protection from many infections as well as from malignant transformation. ${ }^{1-3}$ Mounting evidence suggests that their phenotypic composition and heterogeneity contribute to their function in each context. Based on the expression of receptors CCR7 and CD62L and their associated homing characteristics, memory CD8+ T-cells have been subdivided into two subsets of circulating memory: central memory $(\mathrm{Tcm})$ express the lymph node homing receptors CCR7 and CD62L and maintain a high proliferative capacity, while effector memory T-cells (Tem) lose the expression of these receptors along with proliferative capacity, but acquire increased cytotoxic potential. ${ }^{4}$

More recently, a new category of memory CD8 + T-cells has been characterized in non-lymphoid and lymphoid tissues of both mice and humans and termed resident memory T-cells (Trm). ${ }^{5-13}$ Like Tem, Trm do not express either of the lymph node homing receptors and are thought to reside permanently in the tissue, where they acquire a unique phenotype and gene expression profile. One marker commonly used to define Trm is CD69, which has previously been utilized to identify recently activated lymphocytes in the blood. In tissues, an increase in CD69 expression is related to the downregulation of the transcription factor KLF2, which controls transcription of the receptors for sphingosine-1-phosphate, a molecule involved in chemotactic exit of the cells from the tissue. ${ }^{14,15}$ CD69 is upregulated by type I interferons, IL-33, TNF-a and other cytokines. ${ }^{16-19}$ Another key molecule used to define CD8+ Trm is the integrin alpha $\mathrm{E}$, also known as CD103, which has been shown to be increased by TGF- $\beta .^{19}$ The use of these markers, initially identified in mouse models, allowed for the characterization of human Trm in different tissues, including mucosal, lymphoid, and peripheral non-lymphoid tissue. ${ }^{7,20-23}$ Human Trm upregulate markers such as CD49a, associated with a cytotoxic phenotype, ${ }^{24}$ the inhibitory molecule CD101, and PD1, a molecule associated with exhaustion. ${ }^{21}$ Upon stimulation, Trm can produce the pro-inflammatory cytokines IFN- $\gamma$, IL-2 and IL-17, as well as the immunomodulatory IL-10. ${ }^{21}$

The mucosa lining the female cervicovaginal tract (CVT) represents the entry site for numerous pathogens of high public health importance such as Herpes Simplex virus-2 (HSV-2), human immunodeficiency virus-1 (HIV-1), and human papillomavirus (HPV). Interestingly, there is epidemiological evidence indicating that infection with one pathogen is associated with an increased risk of acquiring subsequent infections, as described for HSV-2 and HIV-1. ${ }^{25-27}$ Further, it has been proposed that inflammation in the CVT, due to pathogens or to dysbiosis of the resident flora, can facilitate subsequent viral infections. ${ }^{28,29}$ In addition, high levels of genital tract inflammation have been correlated to higher HIV set point and shedding. ${ }^{30,31}$ However, it remains unclear which cells contribute to an overall long-term increase in inflammation in the CVT during chronic infections. Furthermore, it is possible that mucosal T-cells could participate in active immunity against chronic infections of the CVT in addition to mediating chronic inflammation. ${ }^{32}$ Thus, we sought to determine if inflammation is driven by a specific subset of mucosal CD8 T-cells or rather by an overall change in the entire CD8 T-cell compartment in the CVT.

We examined the CVT T-cell compartment in healthy women as well as in the context of chronic HIV infection. We used CD69 and CD103 to distinguish the three main subsets of CVT CD8+ T-cells: the double positive CD69+ CD103+ cells (DP), the single positive

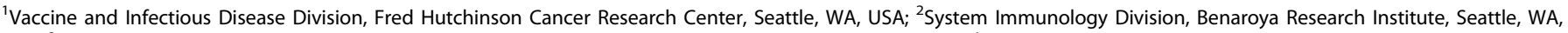

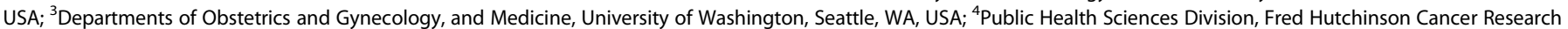
Center, Seattle, WA, USA and ${ }^{5}$ Department of Global Health, University of Washington, Seattle, WA, USA

Correspondence: Jennifer M. Lund (jlund@fredhutch.org)
}

Received: 11 March 2019 Revised: 11 June 2019 Accepted: 14 June 2019

Published online: 16 July 2019 
CD69+ cells (CD69+SP), and the double negative CD69- CD103cells (DN). We found that the first two share characteristics previously described for Trm in non-inflamed lungs and spleen, ${ }^{21}$ but also present some distinctive features, such as the lack of transcriptional up-regulation of the $I L-17$ gene. The DN population of CD8+ memory T-cells in the CVT possesses enhanced cytotoxic and inflammatory potential, and a reduced migratory capacity as compared to their blood counterpart. These DN cells, which we term inflammatory mucosal T-cells (Tim), expand in the CVT of HIV-infected as compared to HIV-uninfected women. Together, these data suggest that inflammatory changes in the CVT of women with chronic infections are not solely driven by changes in the long-lived Trm compartment, but instead by the previously unappreciated Tim subset. Further, it is possible that therapeutic targeting of these CD8 T-cells in the CVT could help restore tissue homeostasis in the context of chronic infection and inflammation.

\section{RESULTS}

CD69 and CD103 identify three populations of T-cells in the CVT that are distinct from circulating T-cells

To identify the differences in CD8 + T-cells from the CVT compared to those from the circulation, we characterized the CD8+ T-cells in vaginal, endocervical, and ectocervical tissues and blood from healthy donors in the Seattle Surgical Cohort. First, we examined the phenotypic profile of CD45 + CD3 + T-cells by high-parameter flow cytometry using a panel including markers of activation, tissue residency, maturation, chemokine expression, and exhaustion (the gating scheme is shown in Supplementary Fig. 1). Dimensionality reduction using t-stochastic neighbor embedding (t-SNE) showed separation between blood and CVT CD8+ and CD4+ T-cells (Fig. 1a). Upon examination of the main surface markers differentiating blood and CVT T-cells, we observed that, as expected, putative Trm markers CD69 and CD103 were more highly expressed in the tissue (Fig. 1b, c). Additionally, CD49a, CD101, PD-1, and CCR5 were also higher in CVT tissue compared to blood T-cells, while the expression of CD25, CD38, and HLA-DR was uniformly low on tissue T-cells and expression of CD127 is comparable to the blood (Fig. 1b, c). Given that CD69 and CD103 have previously been identified as markers of tissue residency, particularly for CD8+ T-cells, ${ }^{5,11,21,33-35}$ we next measured the frequency of CD69-CD103- (double negative; DN), CD69+ CD103$(\mathrm{CD} 69+\mathrm{SP})$, and CD69+ CD103+ (double positive; DP) CD8+Tcell subsets in tissues and blood. We observed that the CD69+ CD103+ fraction was predominant in the CVT tissue regardless of type, though the $\mathrm{DN}$ and $\mathrm{CD} 69+\mathrm{SP}$ populations were detectable (Fig. 1d).

To further compare circulating and CVT T-cells as well as the three CVT CD8+ T-cell subsets distinguished by CD69 and CD103 expression, we evaluated their transcriptional profiles by RNA sequencing. As the vast majority of tissue T cells have an effector memory phenotype, characterized by the lack of CCR7 expression, we excluded CCR7+ populations in the four tissue compartments, including blood, before sorting the three populations based on CD69 and CD103 expression. Specifically, we bulk-sorted the three main populations in the tissues and the two main populations in the blood of four women belonging to the Seattle Surgery Cohort based on CCR7, CD69, and CD103 expression for RNA sequencing (gating and sorting schemes for tissue and blood are shown in Fig. 1e). Multidimensional scaling (MDS) was performed on all of the sorted populations that passed the quality control and the results are shown in Fig. If. Tissue-derived cells were separated from circulating cells by dim1. Further, tissue location was the main driver of differences in gene expression, with a minor component coming from CVT tissue type (vagina, endocervix, and ectocervix) or subset within tissue. Altogether, our results show that T-cells within the CVT have a distinct phenotypic and transcriptional profile compared to circulating T-cells, and that CD69 and CD103 can be used to define three distinct populations of CD8+ T-cells within the CVT.

CD8 + T-cells in the vagina, ectocervix, and endocervix share similar phenotypic and transcriptional patterns

The RNAseq results of blood and tissue CD8+ T-cells (Fig. 1f) prompted us to more thoroughly examine the differences in CD8+ T-cell phenotype between the three CVT tissue types. First, we compared the frequencies of CD4+ and CD8+ T-cells in the three tissues and did not observe any significant differences (Fig. 2a). We then quantified the frequencies of activated $(C D 127$ dim $\mathrm{CD} 25+)$, naive (CCR7+ CD45RA+), central memory (Tcm, CCR7 + CD45RA-) and effector memory (Tem, CCR7-) CD8+ T-cells in each tissue type. As shown in Fig. $2 b$, no differences were observed in frequency of CD8+ T-cell types between endocervical, ectocervical, and vaginal tissues, though as expected, most tissue CD8+ T-cells showed an effector memory phenotype regardless of tissue type. To more thoroughly characterize DN, DP, and CD69+SP CD8+ T-cells, we compared the frequencies of cells expressing the previously described markers of tissue residency CCR5, CXCR3, CD49a, and PD-1 (Fig. 2c). No differences between the three tissues were detectable for any of the analyzed parameters. Additionally, when we examined gene expression profiles based on CVT tissue type, we failed to observe distinct patterns for the three CVT tissues (Fig. 2d). Overall, our results suggest similarity in the phenotype and transcriptional pattern of the CD8+ T-cell compartment within vaginal, endocervical and ectocervical tissues.

Mucosal tissue CD69- CD103-CD8+ T-cells within the CVT are a subset distinct from the circulation

Given the similarities we found between CD8+ T-cells within different CVT tissue sites, we next focused on vaginal tissue as representative of the CVT to comprehensively characterize the three populations of tissue CD8+ T-cells identified by the expression of CD69 and CD103. The CD69- CD103- DN population of CD8+T-cells in human tissue has not been well characterized, and therefore we sought to determine if these cells constitute a unique population or are transient blood cells, similarly to what has been reported for other tissues. ${ }^{21}$ Thus, we sought to further interrogate the gene expression patterns of CD103- CD69- DN CD8+ T-cells in tissue and blood. We focused on genes differentially expressed by tissue DP cells as compared to blood DN CD8 + cells. This gene set comparison was selected in order to identify a tissue signature so that we could next evaluate whether the DN cells have more in common with tissue CD8+ $\mathrm{T}$ cells or with blood CD8 $+\mathrm{T}$ cells. We predicted that if the DN cells in the tissue were blood contaminants that were passing through the tissue at the time of sample collection, they would have a similar expression pattern to blood CD8 $+T$ cells and thus no tissue signature. However, we found that tissue DN cells differed from their blood counterparts, with 61 genes higher in tissue DN and 29 higher in blood DN (Fig. 3a, b). Among the differentially regulated genes, we observed higher expression of genes in tissue DN cells encoding for the chemokines CCL3, CCL4 and CCL4L2, for the chemokine receptor CXCR4, and for IFN- $\gamma$ (Fig. $3 \mathrm{~b})$. The genes upregulated by the DN population in the tissue compared to the blood included a set of genes known for their role in immune functions. Using the STRING database, we identified several pathways enriched in our gene set of upregulated genes: cell migration (ID GO:0016477), localization (GO:0051179) and inflammatory response (GO:0006954) pathways, which include the chemokine-encoding genes CCL3, CCL4, and $C C L 4 L 2$, the chemokine receptor CXCR4, ANXA1 and CD44, while IFN $-\gamma$ belongs to the inflammatory response pathway only (Fig. $3 \mathrm{C}$ ). All three pathways were enriched in our gene set, with a false discovery rate of 0.002 for cell migration (11 genes of the 
a

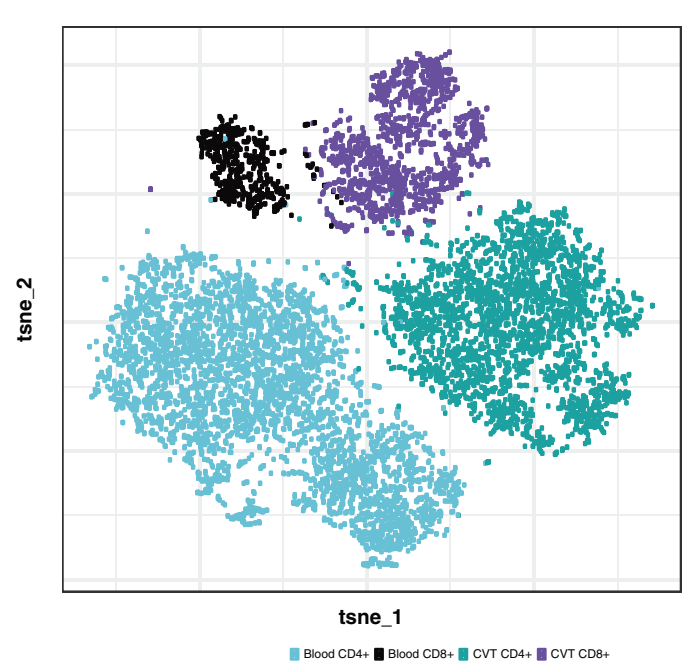

c

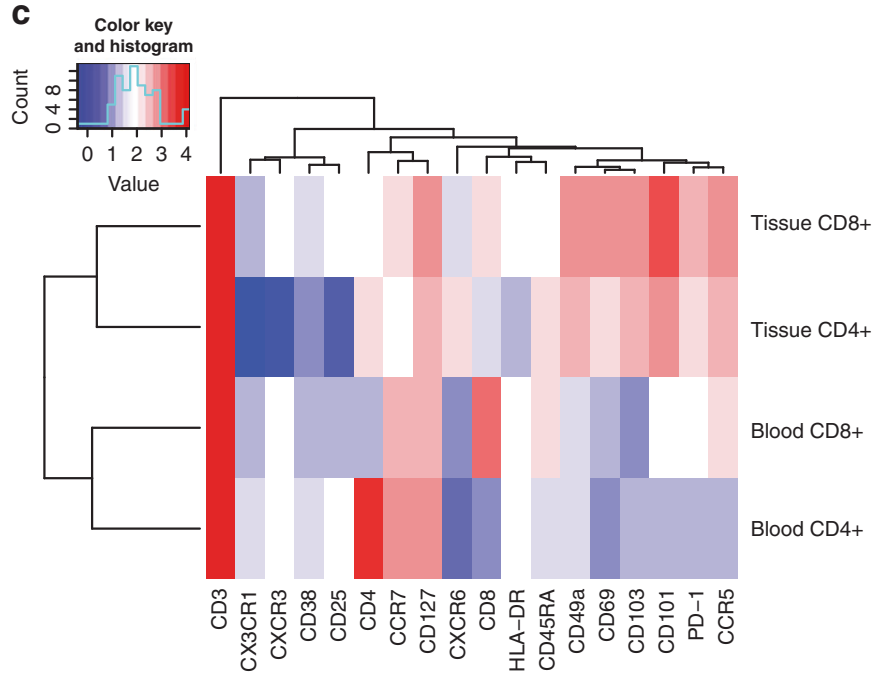

b

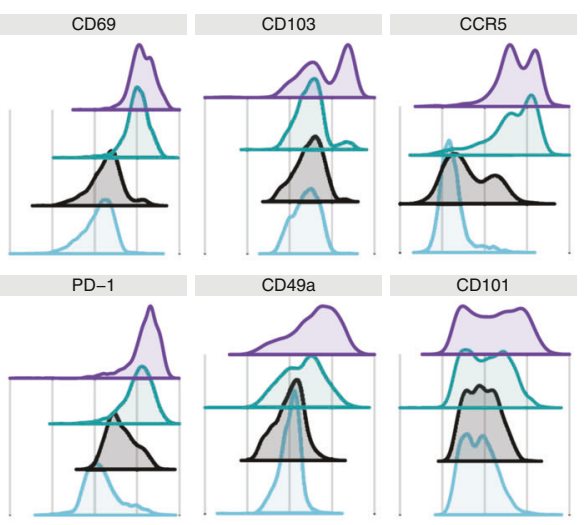

d

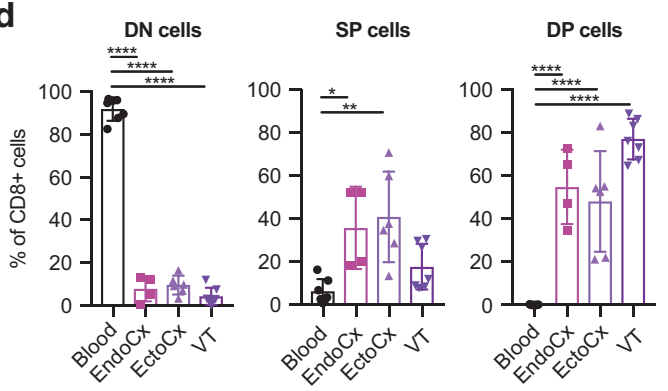

e
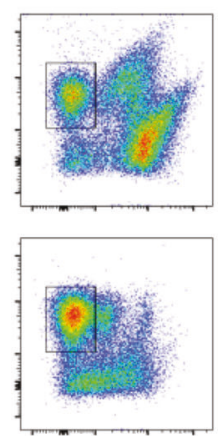

Qิ

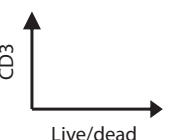

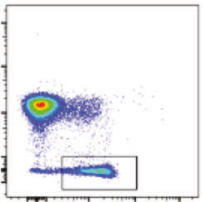
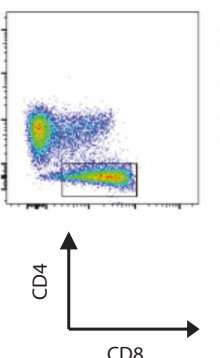
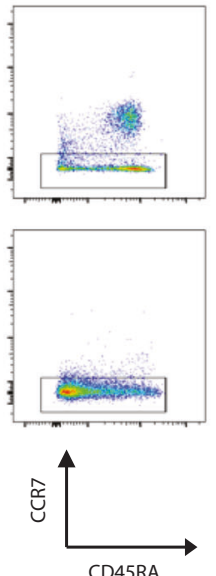
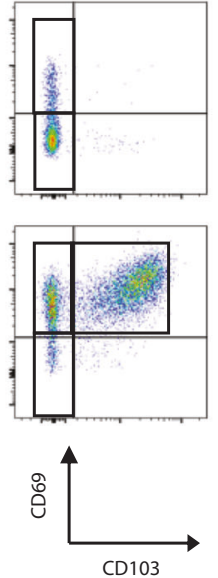

f

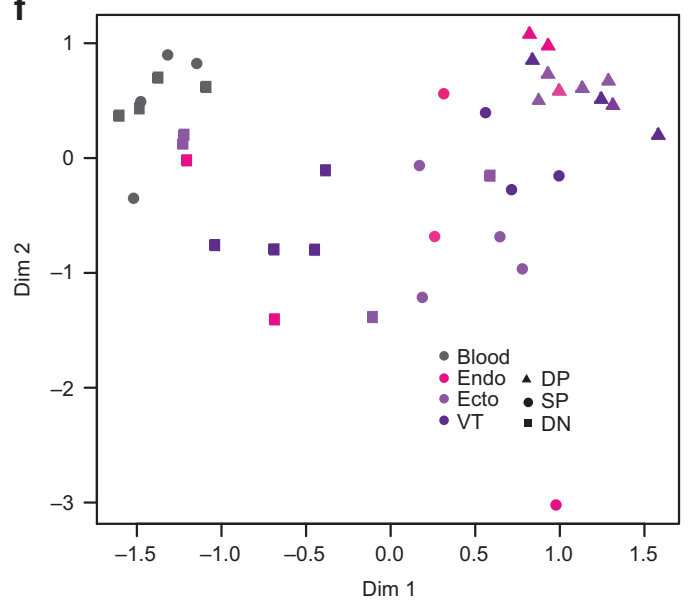

Fig. 1 CD8+T-cells in the blood versus the CVT show phenotypical and transcriptional differences. a Dimensionality reduction of multiple parameters measured by flow cytometric staining in CVT tissues and blood was visualized by a t-SNE plot. Representative histograms (b) and protein expression (determined by flow cytometric staining) heat map (c) show that CD4+and CD8+T-cells in the CVT express high levels of CD69, CD103, CD49a, PD-1, CD101, and CCR5. d Mean frequencies ( \pm SD) of DN, CD69+ and DP cells in blood (black), endocervix (pink), ectocervix (purple) and vaginal tissue (dark purple). Statistical significance was determined by Ordinary one-way ANOVA with Tukey's multiple comparisons test. $n=7,{ }^{*} p<0.05,{ }^{* *} p<0.01,{ }^{* * *} p<0.001$, and ${ }^{* * * *} p<0.0001$. e Gating scheme used for sorting cells in blood (upper panel) and tissues (lower panel). f MDS plot of RNA sequencing data from CD69+CD103+ (DP; triangles), CD69+CD103- (SP; circles), and CD69-CD103- (DN; squares) CD8+ T-cells sorted from the ectocervix, endocervix, blood, or vagina 

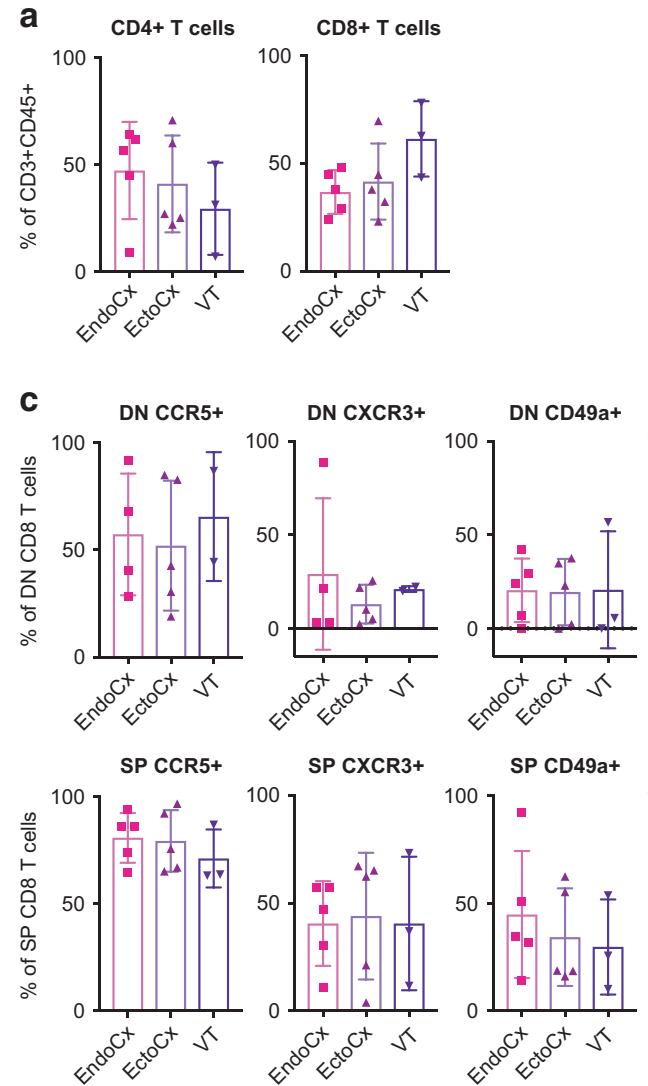

SP CXCR3+
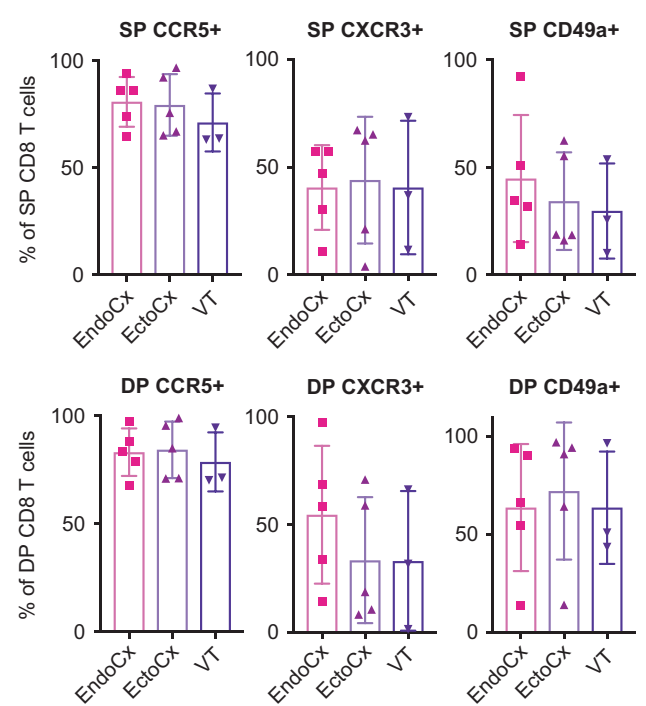
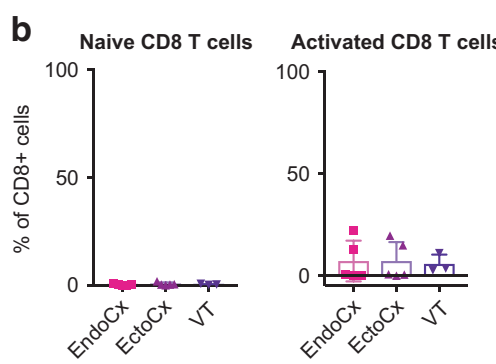

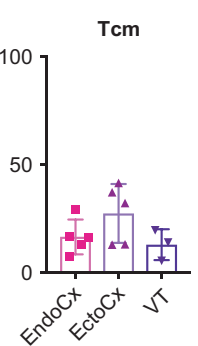

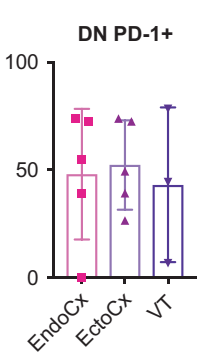

d
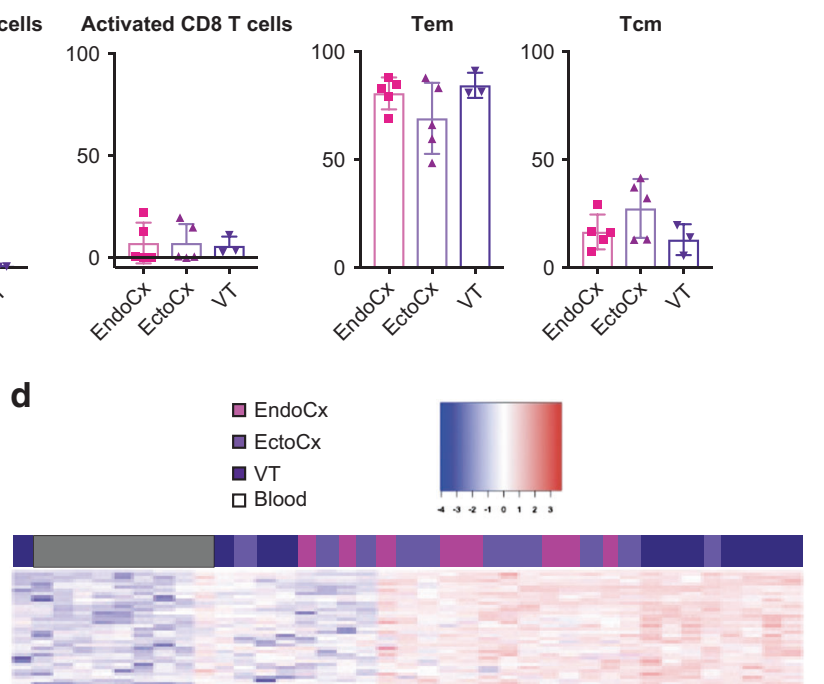

SP PD-1+
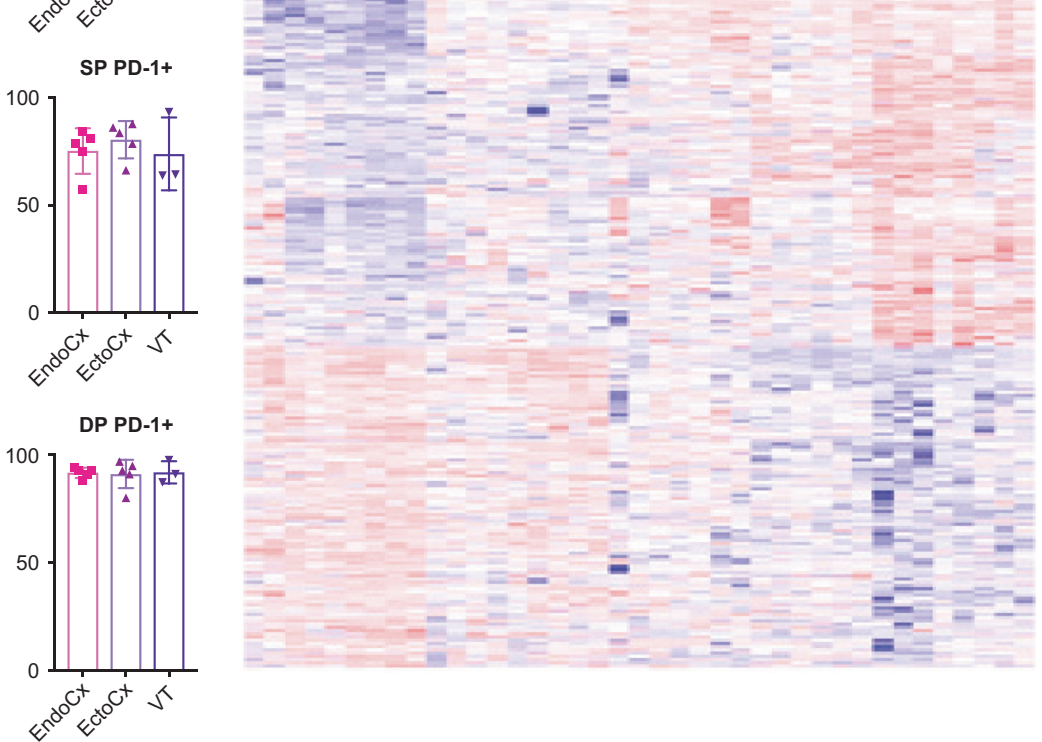

Fig. 2 T-cells in the endocervix, ectocervix and vaginal tissue display similar phenotypes and transcription patterns. a Mean frequencies ( \pm SD) of CD4+ and CD8+ T-cells expressed as percentages of CD3+CD45+ live cells in endocervical (EndoCx), ectocervical (EctoCx) and vaginal tissue (VT) were determined by flow cytometric staining. b Mean frequencies ( \pm SD) of naïve (CCR7+ CD45RA+), activated (CD127dim CD25+), effector memory (Tem, CCR7-) and central memory (Tcm, CCR7+ CD45RA-) CD8+ T-cells in the 3 CVT tissues. c Mean frequencies ( \pm SD) of CCR5, CXCR3, CD49a, and PD-1 as percentages of DN (top), CD69+ (middle) and CD69+CD103+DP (bottom) T-cells for EndoCx, EctoCx and VT. $n=5$. To test for statistical significance, a Kruskal-Wallis test was performed with Dunn's multiple comparisons test. d Heatmap showing the RNA expression patterns of DP, SP, and DN CD8+ T-cells within the blood and the three types of CVT tissues

upregulated genes), 0.03 for localization ( 23 genes) and 0.005 for the inflammatory response (8 genes).

Since we observed the enrichment of cell migration and localization pathways, we next focused our attention on molecules involved in cell migration out of the tissue. We found that RGS1, whose expression has been shown to reduce T-cell migration through modulating the $\mathrm{G}$ protein signaling coupled to chemokine receptors, ${ }^{36}$ was fourfold higher in DN tissue CD8+ T-cells compared to their blood counterparts $(p=0.01)$. In the vaginal tissue, RGS1 was expressed less in the DN cells than the DP cells $(-1.06 \log 2$-scale, $p=0.02)$ or the CD69+ SP populations in the tissue $(-1.11 \log 2$-scale, $p=0.06)$. Given this expression pattern, we hypothesized that the elevated expression of RGS1 would override other migratory signals, thereby reducing chemotaxis in response to G-coupled receptors-binding chemokines, such as CCL19 and CCL21 and S1P. To test this hypothesis, we performed a chemotaxis assay in response to S1P, CCL19, and CCL21, and we observed that the migration capacity of the tissue DN CD8+
T-cells was lower than that of the CD8+ T-cells from the circulation (Fig. 3d). Altogether, given their reduced response to migratory signals despite the lack of canonical Trm markers CD69 and CD103, and their high expression of chemokine receptors as compared to the blood, we define the tissue DN CD8+ T-cell population as a unique and distinct subset from cells of the same phenotype in the blood.

To understand which signals might be driving these DN CD8 T-cells to the CVT mucosa, we measured soluble chemokines and cytokines in cervicovaginal secretions. We quantified 10 chemokines (Table 1) in cervicovaginal fluid collected from five healthy women and evaluated whether there were any correlations with the frequency of the double negative population in the vaginal tissue from the same donors. We observed a positive correlation between the frequency of vaginal DN CD8 T-cells and CCL3 (MIP$1 a, p=0.03)$ as well as IP10 ( $p=0.1$, Fig. 3e). Altogether, our results demonstrate the existence of a CD69-CD103- population of CD8+ T-cells in CVT tissues that is distinct from such cells in the 


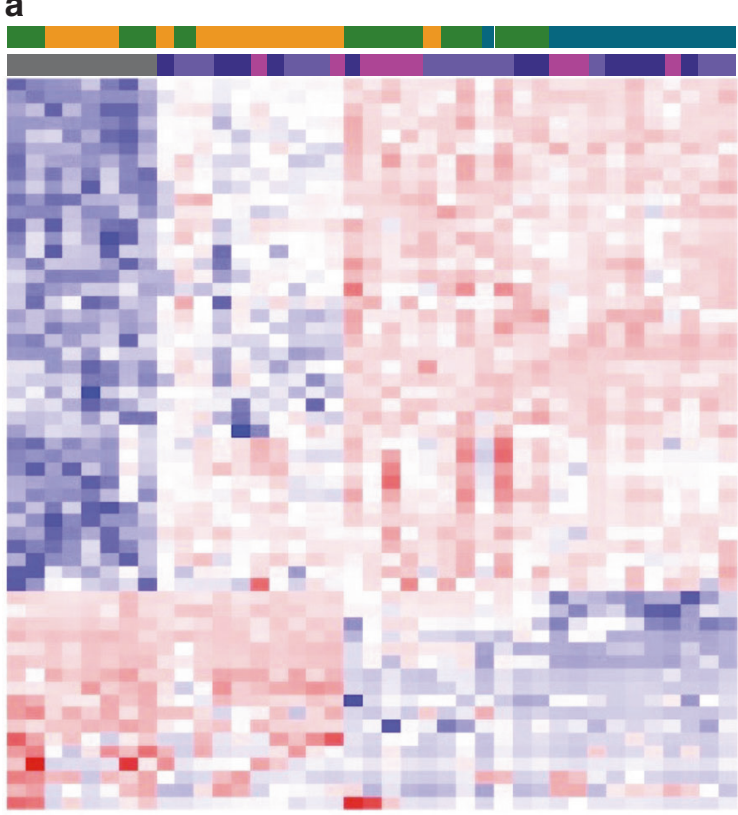

C

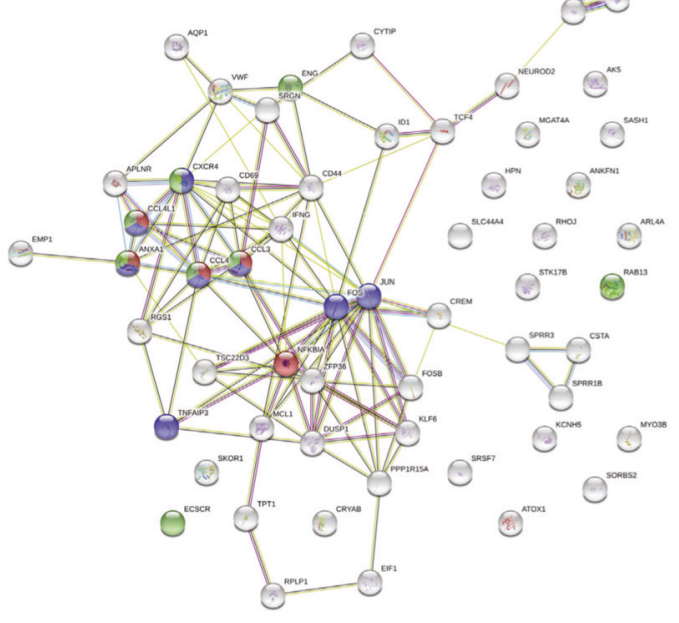

b

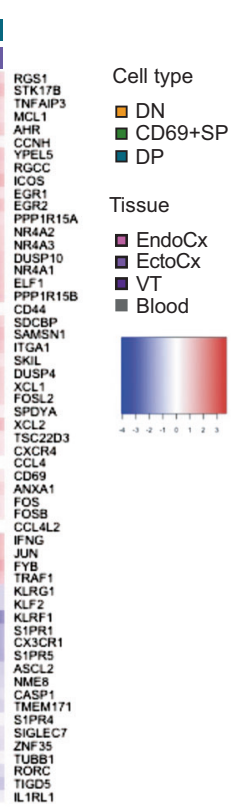

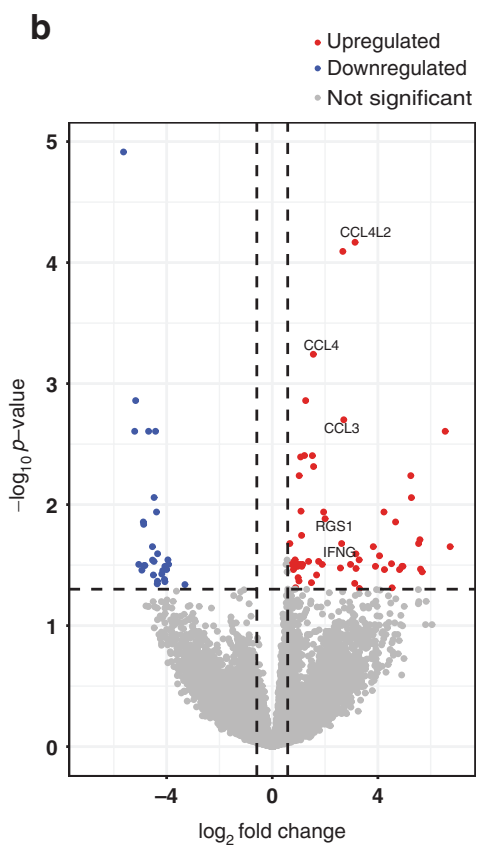

d

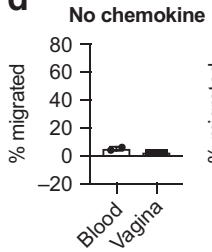

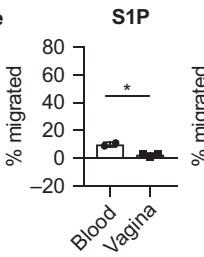
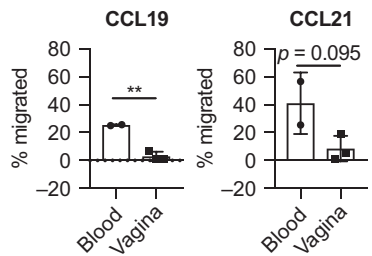

e
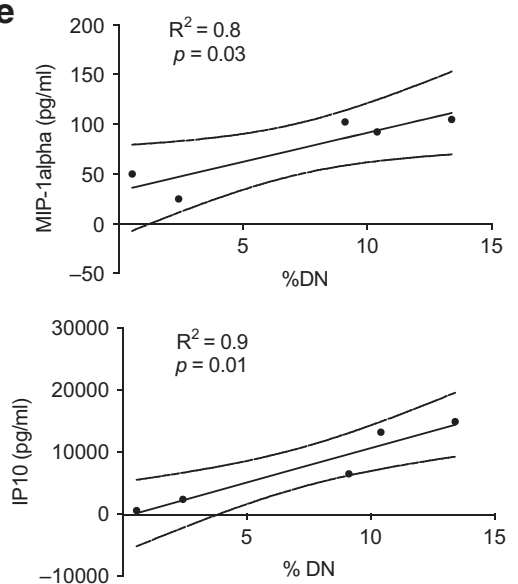

Fig. 3 DN cells in the tissue differ from those in the blood and show a characteristic migration and inflammatory pattern. a Heat map showing the RNA expression pattern of CD8+T-cells for a set of genes differentially expressed in blood DN versus tissue DP cells. $\mathbf{b}$ Volcano plot of genes differentially expressed in CVT tissue DN versus blood DN CD8+T-cells (red = upregulated, blue = downregulated genes). c Pathways enriched in the gene set upregulated in tissue DN versus blood DN. (Red = cell migration pathway, purple = inflammatory pathway, green = localization pathway). d The mean frequencies $( \pm S D)$ of migrated cells as percentages of non-migrated cell in response to S1P, CCL19, and CCL21. Statistical significance was determined by unpaired $t$-test. $n=3$ donors, ${ }^{*} p<0.05,{ }^{*} p<0.01$. e Pearson correlations between the frequencies of DN cells in the vagina and the concentrations of MIP-1a (top panel) and IP10 (bottom panel) in the paired cervicovaginal secretions. Pearson correlation $\left(R^{2}\right)$ and $p$ value are indicated in the graph

circulation. We therefore term this population inflammatory mucosal T-cells, Tim, as they are present in mucosal tissue but do not express the putative Trm markers CD69 or CD103. Further, these tissue cells possess a higher inflammatory potential along with a reduced capacity to follow chemotactic cues to migrate out of the tissue as compared to phenotypically matched, CD69 - CD103-CD8+ T-cells within the circulation.
Tim cells show distinctive transcriptional, phenotypical, and functional characteristics compared to other tissue CD8+ T-cell subsets

To determine if Tim are phenotypically and functionally distinct from other tissue CD8 + T-cells within the CVT, we next evaluated the transcriptional differences between Tim and DP Trm-like cells. We found 120 downregulated and 103 upregulated genes (Fig. 4a). 
Table 1. Cytokine and chemokine plating scheme for the MSD analyses

\begin{tabular}{lllll}
\hline Plate 1 & Plate 2 & Plate 3 & Plate 4 & Plate 5 \\
\hline IFN- $\gamma$ & IP-10 & IL-17F & MIP-5 & TGF- $\beta 1$ \\
IL-1 $\beta$ & MCP-1 & IL-21 & & \\
IL-2 & MCP-4 & IL-22 & & TGF- $\beta 2$ \\
IL-4 & MIP-1 $\alpha$ & IL-23 & & \\
IL-5 & MIP-1 $\beta$ & IL-31 & & \\
IL-8 & IFN- $\alpha 2 A$ & IL-33 & & \\
IL-10 & IL-1 $\alpha$ & IFN- $\beta$ & & \\
IL-12p70 & IL-15 & IL-1R $\alpha$ & & \\
IL-17A & IL-18 & IL-2R $\alpha$ & & \\
MIP-3 $\alpha$ & MIP-3 3 & SDF-1a & MCP-2 & TGF- $\beta 3$ \\
\hline
\end{tabular}

Compared to DP CD8+ T-cells in the CVT, we detected the upregulation of genes required for cells to exit the tissue in Tim cells, such as the S1P receptors S1PR1 and S1PR5 and their transcription factor KLF2. Moreover, we observed altered expression of RAP1GAP2 and $C X 3 C R 1$, which have previously been reported to be involved in tissue retention, and of CD101, an inhibitory molecule highly expressed on Trm. ${ }^{21,37}$ Interestingly, we found the enrichment of the TCR pathway in the downregulated gene set $(p=0.00007)$.

When we compared genes differentially expressed by Tim compared to the CD69+ CD8+ T-cells, we identified only 19 upmodulated and 35 downmodulated genes (Fig. 4b). Similar to the comparison of Tim to DP CD8+ T-cells, we also found a lower CX3CR1 expression, but we did not see any differences with regards to the S1P receptor-encoding genes in Tim vs CD69 SP CD8+ T-cells. However, we identified a significant downmodulation of genes belonging to the TCR pathway compared to CD69 SP CD8+ T-cells (0.01). Therefore, Tim do not appear to be actively receiving a TCR stimulation; moreover, they do not display a gene expression signature consistent with a memory phenotype. In fact, they express higher levels of KLRG1 and lower levels of CD44 as compared to both the CD69+SP and DP populations (Fig. 4a, b).

Next, we compared the phenotype of the three CD8+ T-cell populations in the vaginal tissue by flow cytometry, with a focus on markers identified by RNA sequencing or previously described as signature molecules for Trm, such as CD49a (ITGA1), CD101, CCR5, and PD-1. We confirmed that lower frequencies of DN Tim express CD101, PD-1 and CCR5 as compared to the DP CD8+ Tcell population (Fig. 4c), whereas we failed to observe any significant difference in their expression of CXCR3, CX3CR1, and CCR6, as well as in the percentages of activated cells defined as CD127dim CD25+ and HLA-DR+CD38+ (Supplementary Fig. 2). When we examined the distribution of classically-defined CD8 Tcell subsets within the 3 populations, we observed that a higher percentage of vaginal DN Tim cells are CCR7- CD45RA+, or TEMRA phenotype cells, as compared to double positive vaginal CD8 T-cells. Conversely, fewer vaginal DN cells display a Tem phenotype compared to DP tissue CD8 T-cells (Fig. 4d).

Next, we sought to determine if Tim cells have functional differences as compared to CD69+ or DP vaginal tissue CD8+ Tcell populations. Thus, we quantified the percentages of cells secreting granzyme B (GZB-intermediate, or GZB-int), IFN- $\gamma$, TNF- $a$, and TGF- $\beta$ after stimulation. There were no significant differences in the frequencies of the three $\mathrm{CD} 8+\mathrm{T}$-cell populations that produced each of these molecules (Fig. 4e). Strikingly, however, Tim cells appeared to produce higher concentrations of granzyme $B$, resulting in the presence of a unique granzyme B-high (GZB-hi) population present only within the DN Tim subset (Fig. 4e, f). Taken together, our results indicate that vaginal Tim cells are transcriptionally and phenotypically distinct from tissue CD69+ SP and DP cells that have been considered to be Trm-like cells. Moreover, there appears to be a gradient of various factors that ensure retention in the tissue: while $\operatorname{rgs} 1$ is highly expressed in all subsets, CX3CR1 is added in CD69+ SP and DP, while the S1P receptors are higher compared to the DP population only.

The CD69+ SP and CD69+CD103+ DP populations of vaginal CD8+ T-cells have characteristics consistent with a resident memory program with some distinct tissue features

To determine if the three tissue CD8 + T-cell populations hereby described for the vagina are comparable to those in other tissue sites, we compared gene expression in the two CD69+populations (CD103+ and CD103-) versus the DN population and aligned them to the previously published gene signatures of tissue residency for lymphoid and non-lymphoid tissues. ${ }^{21}$ MDS showed that the two sets of genes overlapped significantly for both up and downregulated genes $\left(p=1.76 \mathrm{e}^{-09}\right.$ and $p=2.65 \mathrm{e}^{-23}$ respectively, Fig. 5a). In particular, both sets highlighted the upmodulation of ITGA1, encoding for the integrin alpha subunit CD49a, and the downmodulation of the genes encoding for CX3CR1, S1PR1, and KLF2 in the CD69+ populations as compared to the DN population.

Interestingly, we identified some differences between the two sets, with 188 genes differentially regulated in the CVT as compared to other tissues including lung and spleen (Fig. 5b). While other tissues show a Tc17 signature in CD69+cells, with the upmodulation of IL-17, IL-17RA, IL-23R, and CCR6, we did not observe this, nor did we detect any IL-17A secretion upon stimulation (Fig. 5c). We also failed to observe any IL-10 upregulation, in contrast with previous observations. Instead, we detected an increase in IL-2, both at the gene expression level as well as at the protein expression level upon stimulation (Fig. 5c). Finally, in our gene set we detected lower expression of KLRG1, which has been shown to be lost in memory T-cells, ${ }^{38}$ as well as higher expression of CD44 by the CD69+ populations. The previous study also found upregulation of the chemokine receptors CXCR6, CCR1 and CXCL13, ${ }^{21}$ but we did not observe the same pattern, which could be due to the migration pattern needed to migrate to tissues other than the CVT.

Of note, when we examined the differences between the DP and the CD69+SP population, we identified few genes differently modulated ( 8 upmodulated and 10 downmodulated genes). While the expression profile is similar for the two CD69-expressing populations in the vaginal tissue, there are some differences of note. KLRG1 was upregulated in CD69+single positive cells, while ITGA1 and ITGAE were downregulated as compared to the DP population (Supplementary Fig. 3).

In sum, we demonstrate that the CVT Trm-like CD8+ T-cells that express CD69 share some phenotypic and transcriptional characteristics with those in other non-lymphoid tissues, but also have some unique features. These include a distinct cytokine secretion profile, which might be required for appropriate immunity at specific tissue sites.

Tim frequency is increased in HIV + women while maintaining a consistent phenotype

Given the distinct local cytokine and chemokine milieu that characterizes chronic infections such as $\mathrm{HIV}^{39-44}$ as well as the potential role that CD8+ T-cells could play in immune-mediated pathogen control and/or inflammation, we next sought to determine whether such change affects the distribution and characteristics of CD8+ T-cells in the CVT. Both HIV and bacterial vaginosis (BV) are associated with increased concentrations of Th1 cytokines, ${ }^{39-44}$ which has not been observed for HSV, ${ }^{45}$ possibly due to the capacity of the virus to decrease the stability of cytokine-encoding RNA. ${ }^{46}$ To understand the effects of infections at the mucosal site on local CD8+ T cells, we compared the 


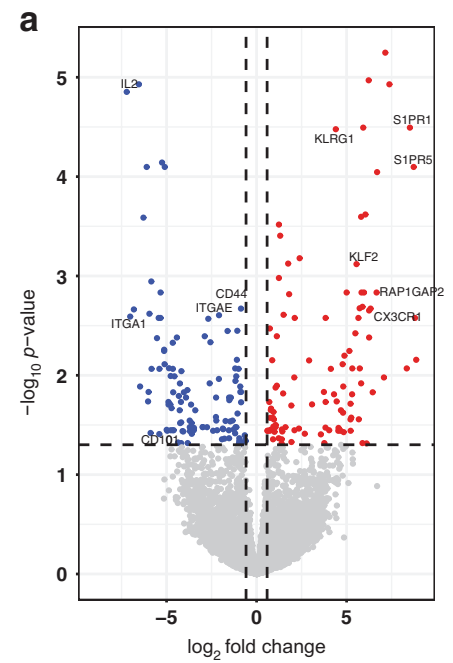

C
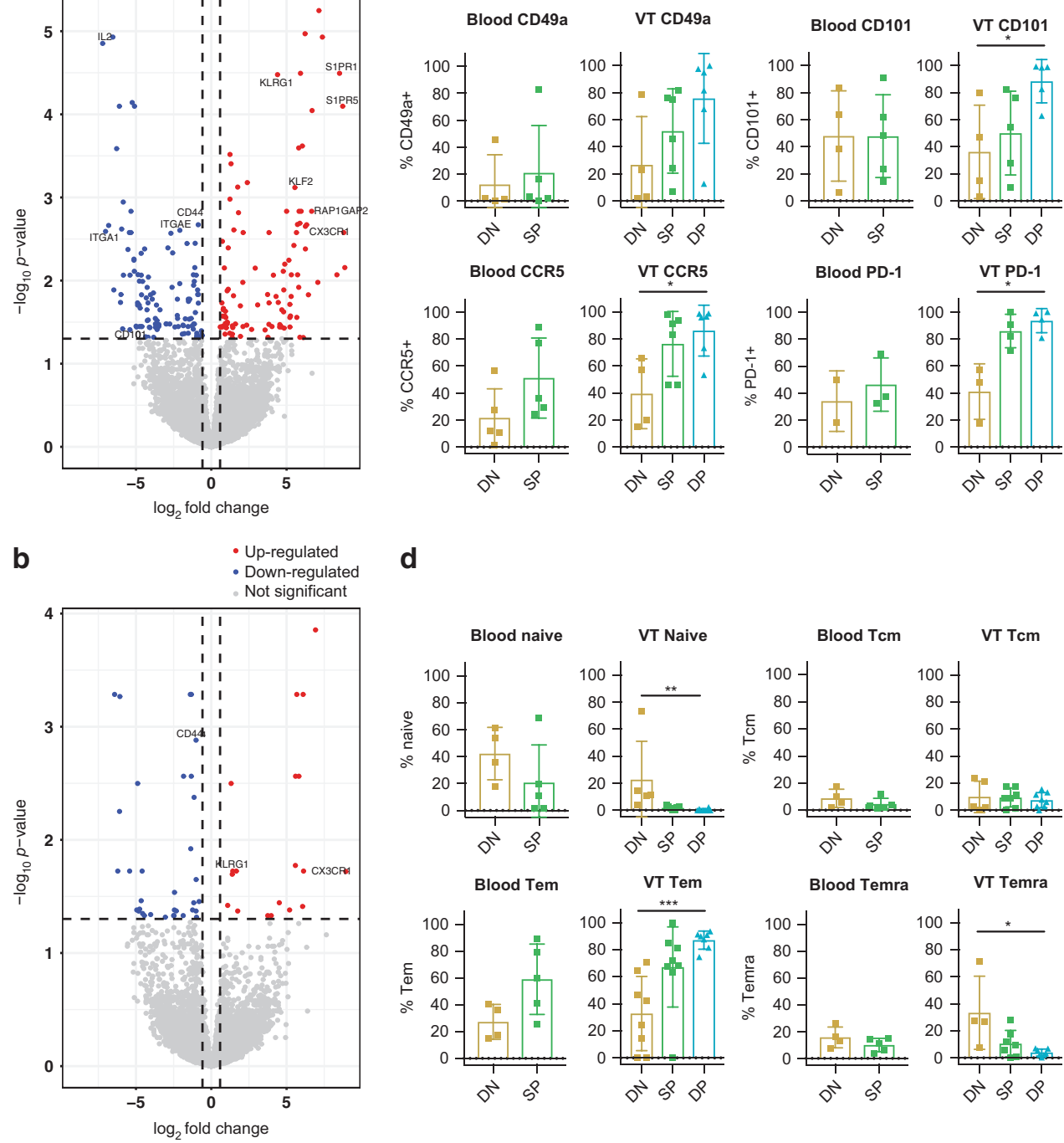

d
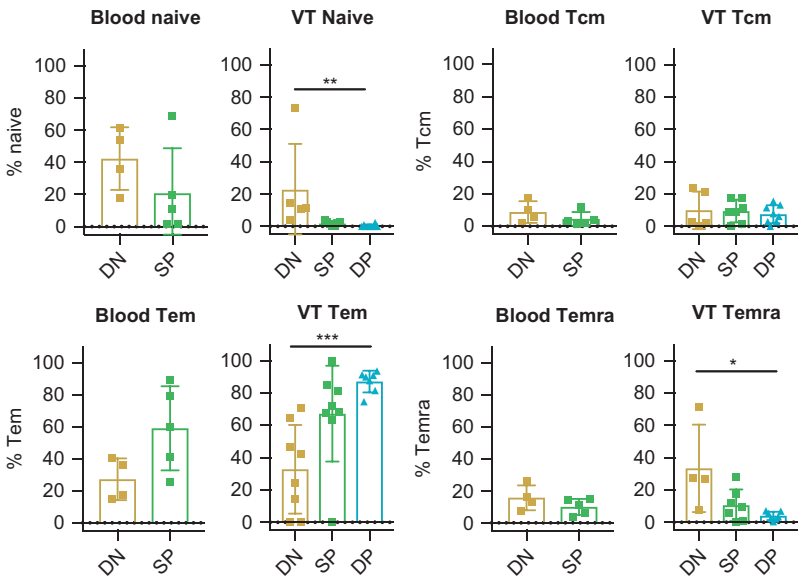

e
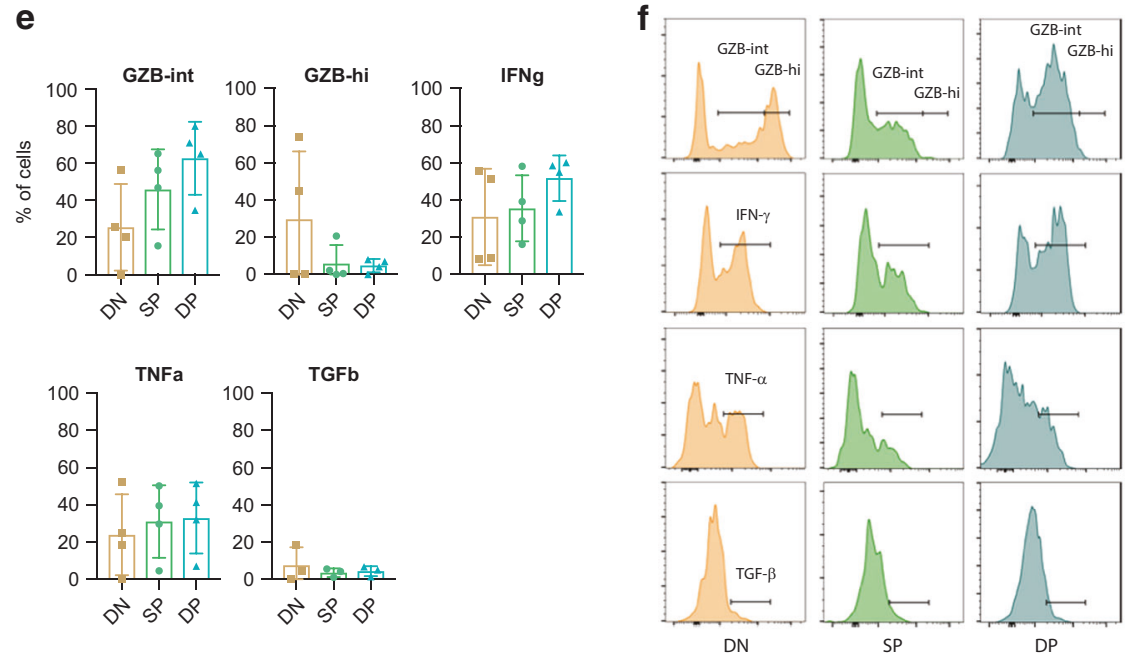

phenotype of tissue CD8 + T-cells in a total of 12 women. Among them, eight are HIV-uninfected and four HIV-infected; of those, three have BV. Finally, five women are HSV-1 or HSV-2 positive and three are negative for both (Table 2 and Supplementary Table 1). Interestingly, we observed a statistically significant increase in the

frequency of the Tim population of CD8 + T-cells within the vagina of HIV-infected versus uninfected women, while the frequencies of CD69+CD103+ and CD69+ cells were not significantly different based on HIV infection status. The difference between groups was further increased when we stratified based on BV status, while HSV 
Fig. 4 DN cells display distinctive transcriptional, phenotypic and functional characteristics as compared to the Trm-like CVT CD8+T-cells. Volcano plot showing the genes differentially regulated in the vaginal DN versus the vaginal DP (a) and the vaginal CD69+SP (b) CD8+T-cell populations. c Histograms showing mean frequencies $( \pm S D)$ of DN, SP, and DP CD8+T-cells expressing CD49a, CD101, CCR5, and PD-1 as determined by flow cytometry. $\mathbf{d}$ Mean frequencies $( \pm S D)$ of the indicated phenotype of CD8+T-cells in blood and vaginal tract (VT) tissue determined by flow cytometry staining. Statistical significance was determined by Mann-Whitney test or a Kruskal-Wallis test with Dunn's multiple comparisons test. $n=5-8,{ }^{*} p<0.05,{ }^{* *} p<0.01,{ }^{* * *} p<0.001$, and ${ }^{* * * *} p<0.0001$. ND non-detectable. e Histogram showing the mean frequencies $( \pm S D)$ of different cytokine-producing DN, CD69+ SP, and DN CD8+ T-cells measured by flow cytometry. To test for statistical significance, a Kruskal-Wallis test was performed with Dunn's multiple comparisons test. f Representative histograms showing granzyme B, IFN- $\gamma$, TNF- $\alpha$, and TGF- $\beta$ production by the three cell types

a

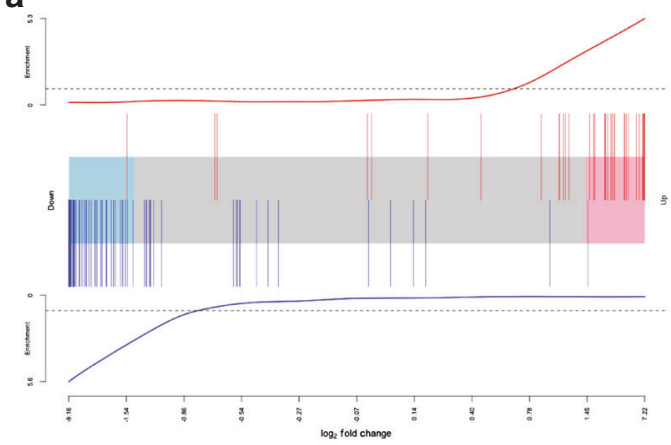

b

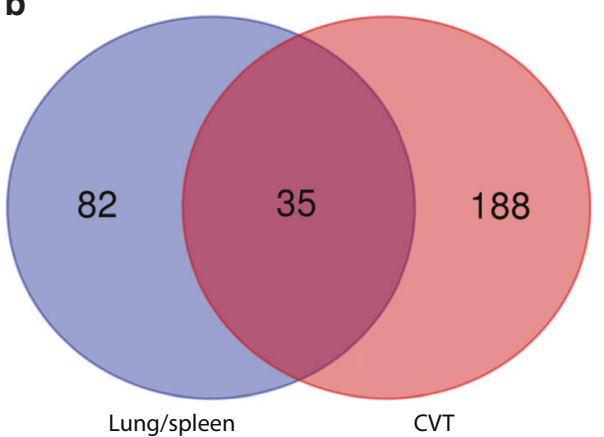

C
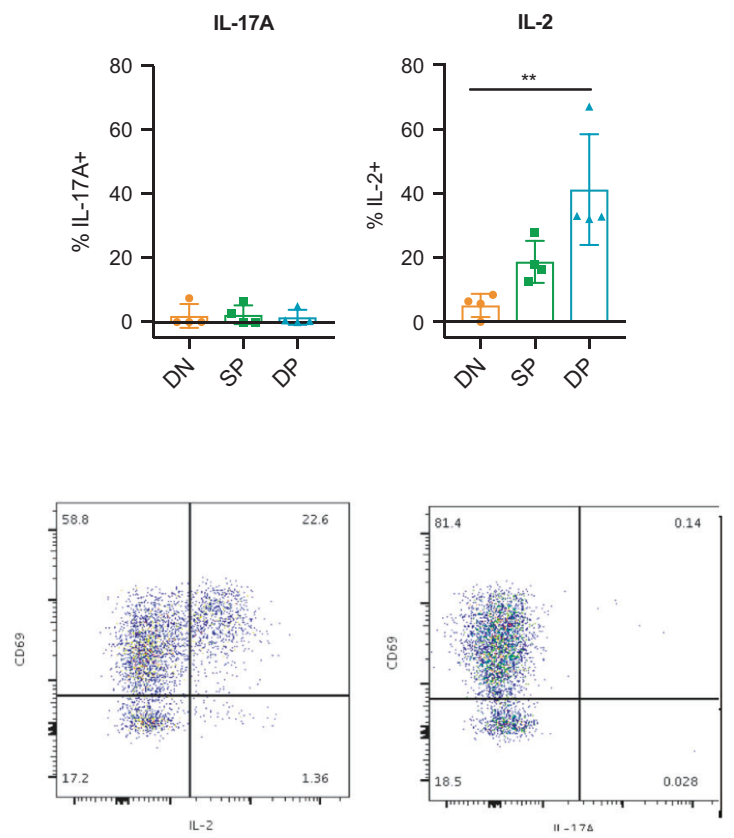

Fig. 5 Cervicovaginal tissue CD8 + T-cells exhibit distinctive features as compared to CD8+ T-cells from other tissues. a Gene set enrichment analyses showing overlap between upregulated (red) and downregulated (blue) genes in the CD69+CD8+ T-cell population as compared to DN CD8+ T-cells in the CVT and in previously published gene sets for lung and spleen. $\mathbf{b}$ Venn diagram showing the numbers of overlapping and non-overlapping genes in the two gene sets analyzed. c Mean frequencies ( \pm SD) (top) and representative plots (bottom) of CD8+ T-cells producing IL-17A and IL-2 upon a 5 h-PMI/ionomycin stimulation as determined by flow cytometry. To test for statistical significance, a Kruskal-Wallis test was performed with Dunn's multiple comparisons test. ${ }^{* *} p<0.01$

infection did not significantly affect Tim frequency (Fig. 6a). Similarly, we detected a higher concentration of the two inflammatory cytokines IL-1a and IL-15 in the cervicovaginal fluids of HIV+ or BV+ women, but not in those of HSV-infected women (Fig. 6b). The increase in DN cells in the context of infection was not associated with a notable alteration in cellular phenotype, as there was no difference in expression of CCR5 based on HIV infection status (Supplementary Fig. 4). Finally, we observed an inverse correlation between the frequency of DN CD8+ tissue Tcells and the concentrations of MIP-1a and IP10 (trend) in the cervicovaginal fluid of HIV+ women (Supplementary Fig. 5), in contrast to what we observed for the HIV - women (Fig. 3e).

Finally, we performed RNA sequencing of the three tissue populations from HIV + and HIV - women. MDS performed on RNA sequencing data from the 3 populations revealed no prominent difference based on HIV infection status (Fig. 6c), and no differentially expressed genes were detected between HIVinfected and uninfected women, as shown in a volcano plot (Fig. 6d).

Altogether, based on our phenotypic and transcriptional characterization of CVT tissue CD8+ T-cells, we propose that Tim in the CVT represent a distinct subset of inflammatory mucosal CD8+ T-cells that reside in tissues through mechanisms distinct from CD69+ or CD69+ CD103+ T-cells. Additionally, they possess distinct functional properties, such as increased proinflammatory and cytotoxic potential, that may endow these cells with the capacity to differentially participate in the anti-pathogen immune responses and/or inflammation.

\section{DISCUSSION}

Several studies have characterized CD8+ T-cells in barrier tissues, ${ }^{21-23,35,47}$ yet there remains a gap in knowledge on those residing in the CVT, which is a biologically relevant tissue site given that it is the portal of entry for numerous pathogens of global health importance. Moreover, inflammation at this site, due to pathogen infection or to alterations within the commensal flora, has been shown to be associated with increased risk of further infections. ${ }^{28,29}$ While mounting evidence support a lead role for resident memory T-cells in inflammation in other tissues, ${ }^{48}$ little is known about T-cells within the human CVT, and in particular those that do not express the putative Trm markers CD69 and/or CD103. In other tissues, the CD69- CD103- population has been shown to consist of blood cells merely passing through rather 
Table 2. Enrollment characteristics of the Seattle HIV Cohort at date of sample collection

\begin{tabular}{llll}
\hline Characteristics & $\begin{array}{l}\text { Median or number } \\
\text { HIV }+(N=5)\end{array}$ & $\begin{array}{l}\text { (Range OR \%) } \\
\text { HIV }-(N=6)\end{array}$ & $P$ Value \\
\hline Age & $40(30-43)$ & $26.7(23-45)$ & $\mathrm{ns}$ \\
Plasma VL & Undetectable & $\mathrm{n} / \mathrm{a}$ & $\mathrm{n} / \mathrm{a}$ \\
CD4+ T-cell count & $407(354-1299)$ & & $\mathrm{n} / \mathrm{a}$ \\
Bacterial vaginosis & $3(60)$ & 0 & $<0.0001$ \\
HSV-2 & $3(60)$ & $1(20)$ & 0.04 \\
HSV-1 & $3(60)$ & 0 & $<0.0001$ \\
Bacterial STD & 0 & 0 & $\mathrm{~ns}$ \\
\hline
\end{tabular}

than tissue-associated cells, ${ }^{21}$ so we first evaluated whether this holds true for the CVT, and we found that the DN population in the CVT is distinct from its blood counterpart. In fact, Tim transcriptional patterns are distinct (Fig. 3), and their migratory capacity in response to the tissue-egress signal S1P and to the lymphoid organ chemotactic molecules CCL19 and CCL21 is reduced as compared to blood DN CD8+ T-cells, while it is comparable to tissue CD69+ T-cells. Upon examination of the expression profile of genes involved in migration, we did not detect any downmodulation of the S1P pathway, nor the upregulation of CX3CR1, two previously described mechanisms for tissue retention of Trm. Interestingly though, we noticed the upregulation of RGS1. RGS1 protein belongs to the family of the regulators of $\mathrm{G}$ protein signaling: it binds to $\mathrm{Ga}$ proteins and increases their GTPase activity, thereby attenuating their signaling. ${ }^{49}$ Chemokine receptors such as CCR7 and S1P receptors are G protein-coupled, and therefore subjected to control by RGS proteins; in particular, RGS1 has been found to reduce $\mathrm{B}^{50}$ and T-cell migration in response to chemotactic stimuli CCL19 and CCL21 in gut tissue. ${ }^{36,51}$ Therefore, a higher expression of RGS1 might be sufficient to reduce the response to chemotactic signals, and the function of other retention signals might be at least in part redundant.

Tim cells also exhibit a particular chemokine transcription pattern; levels of CCL3, CCL4 and CCL4L2 gene expression were increased as compared to the blood DN cells. The chemokines

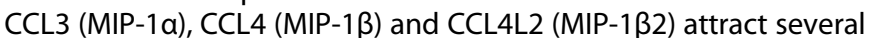
immune cells, including T-cells, monocytes and NK cells by binding CCR1, CCR2, CCR4 and CCR5, and may be poised to participate in local immune responses to infectious challenges, possibly in a bystander-mediated fashion. Notably, CCR5 is one of the co-receptors used by HIV, and MIP-1 $\alpha$ and MIP-1 $\beta$ have been shown to be able to inhibit viral entry. ${ }^{52}$ Thus, in the context of HIV infection, Tim cells could have either a protective or a detrimental function by blocking the CCR5 receptor or by increasing the availability of its target cells, respectively. Moreover, they might fuel inflammation in the CVT of HIV-infected women. Based on their chemokine transcription pattern we termed these CD69CD103 - cells in the CVT inflammatory mucosal T-cells, or Tim, and assessed their frequency in the context of chronic infections. Two previous studies reported an increased frequency of CD103CD8 + T-cells in the female reproductive tract of HIV-infected women $^{53,54}$ and we similarly found the Tim population (which is both CD69- and CD103-) to be expanded in the vaginal tissue of HIV+ women (Fig. 6a). Thus, we evaluated whether this population differed transcriptionally in HIV-infected and uninfected women, but we did not detect any differences in transcriptional patterns in Tim cells from HIV + and HIV - women. We recognize that this could be due to the restricted samples size and therefore to the limited power of the study to detect differences between the populations we compared. Therefore, we cannot exclude that Tim cells, as well as the other populations in the CVT, differ in HIV-infected and uninfected women. Despite the limited sample size, however, we are confident that HIV infection leads to an increase in Tim frequency. Furthermore, when we stratified based on BV status alone, we similarly found that Tim frequency is increased in the CVT of women with BV. It is possible that the two conditions have an additive effect on the distribution of T cells based on their expression of CD69 and CD103. We failed to observe the same statistically significant difference when we subdivided groups based on HSV infection, though this could be due at least in part to the limited sample size, and so further examinations are warranted.

While Tim frequency is low in vaginal tissues from healthy women, their increased abundance in the context of chronic infections suggests a unique role for these cells as compared to other tissue CD8+ T-cell populations. Therefore, we assessed whether Tim are also distinct from the CD69+SP or the CD69+ CD103+ Trm-like cells. Interestingly, when we compared the transcriptional patterns of Tim to either the CD69+ or CD69+ CD103+ CD8 + T-cell populations, we found a significant downregulation of the TCR signaling pathway, and a higher expression of KLRG1. Additionally, upon stimulation, Tim are capable of producing granzyme B in higher concentrations than CD69+ Tcells. Therefore, Tim cells might expand as a result of bystander activation $^{55}$ or at least partially constitute a virtual memory T-cell population ${ }^{56}$ that could participate locally in inflammation. Given the absence of a gene signature indicative of recent proliferation, we speculate that Tim accumulation in the CVT results from a modified chemokine milieu rather than significant in situ proliferation.

To further evaluate how the CD8+ T-cell in the CVT relate to those in other tissue sites, we compared the differences in gene expression between Tim and CD69+ cells with those recently reported for lung and spleen. ${ }^{21}$ We found significant overlap between our data and a previously published gene set (Fig. 5); for example, in the expression of ITGA1 and CD101. However, we also observed some striking differences in our analysis of CVT CD8+ Tcells. First of all, the cytokine signatures of Trm-like cells were distinct in the two studies. While a Tc17 signature of CD69+CD8+ T-cells, together with a high IL-10 expression, have been reported for Trm in other tissues, we failed to observe the same characteristics in the CVT. It has been previously observed that CD8+CD49a- cells are mostly responsible for IL-17 production, while CD49a+ show Tc1 functional characteristics. ${ }^{24}$ In the CVT, the vast majority of DP Trm are CD49a+, which might at least partially explain such differences. Instead, we observed a high production of IL-2 by CD69+ Trm-like cells as compared to the DN population. Therefore, vaginal CD69+ and DP CD8+ T-cells present some key features as compared to similarly-phenotyped cells in other tissues, which might be important for maintaining a balance between immune responsiveness and immune tolerance in this unique tissue site. Our findings thus highlight the potential specialization of immunity within the CVT, which is likely shaped by its particular tissue functions, infectious challenges, and microenvironment that instructs immunity within this tissue site.

Taken together, our results demonstrate the existence within the CVT of a subset of inflammatory mucosal CD8+ T-cells, Tim, that are distinct from both circulating CD8 + T-cells as well as from Trm-like CD8+ T-cells within the CVT. In particular, Tim possess distinct migratory, transcriptional and phenotypic characteristics, including enhanced cytotoxic and inflammatory features. While these cells were located within the CVT at the time of tissue sampling and have properties that suggest reduced capacity for tissue egress, we have not formally demonstrated that these cells are resident in the mucosa, as this is difficult to establish using human tissue samples. In addition, further work is needed to determine if Tim cells are also present within other mucosal tissue 


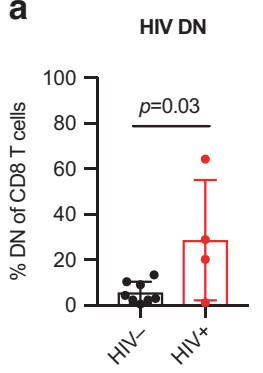

HIV SP
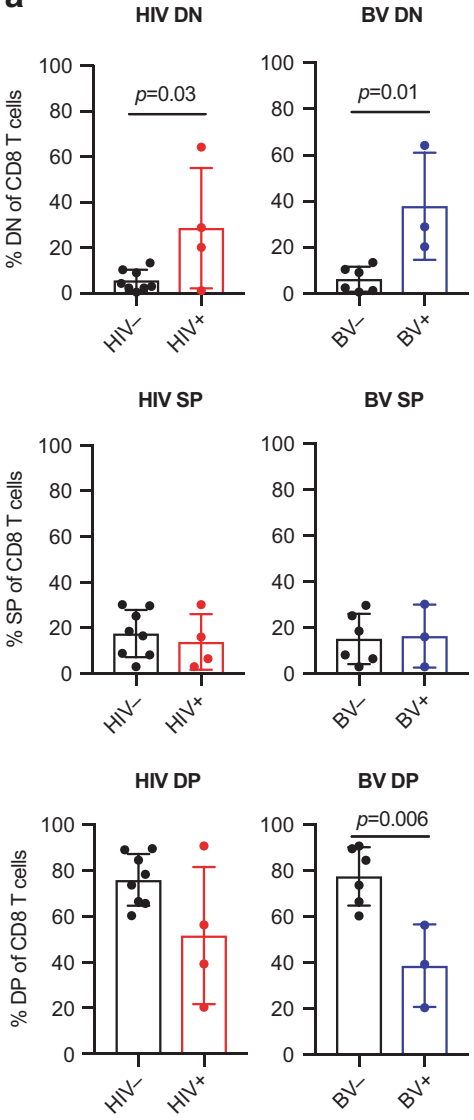

BV SP

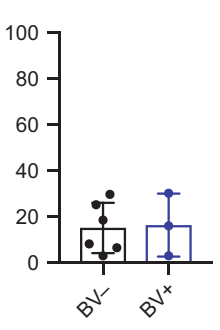

BV DP
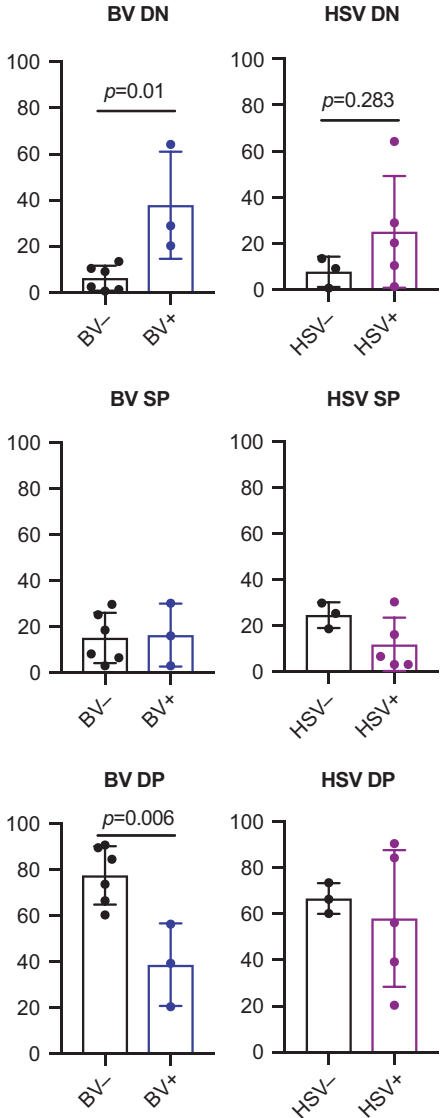

HSV SP

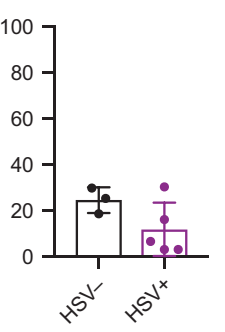

HSV DP

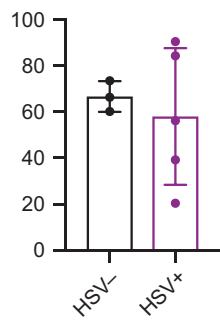

b

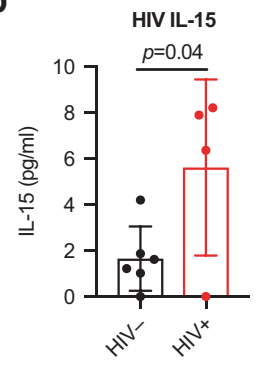

BV IL-15

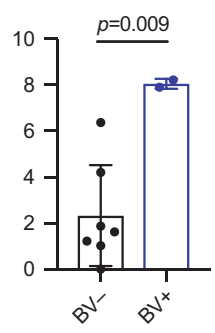

HSV IL-15

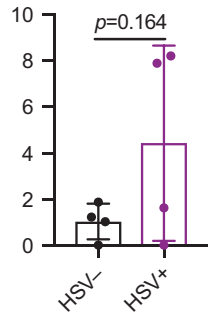

HSV IL-1a
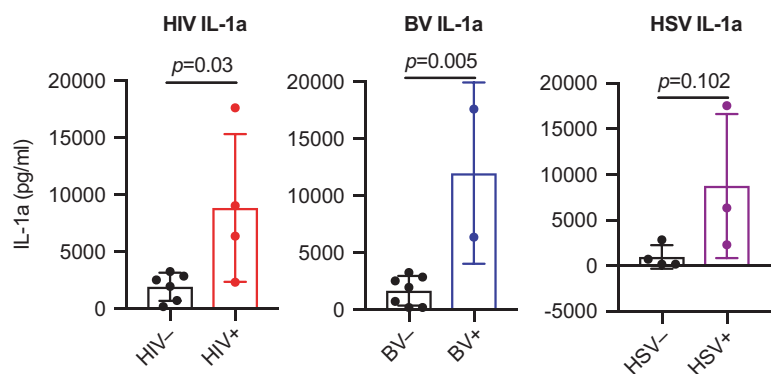

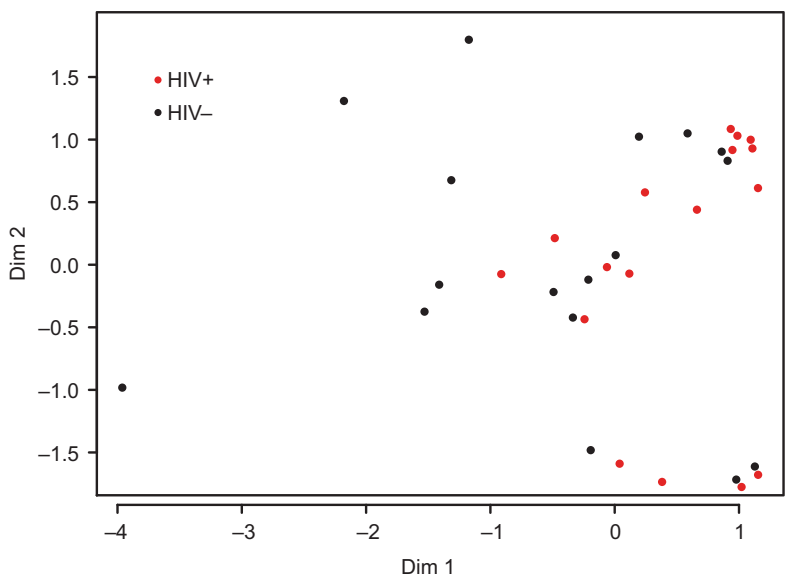

d

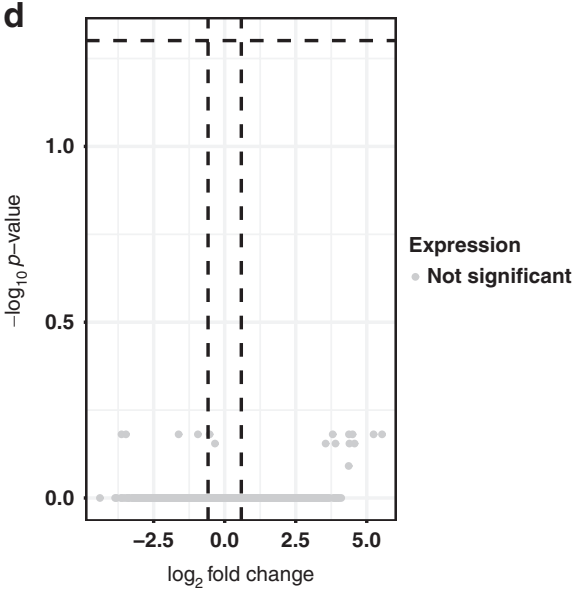

Fig. 6 The frequency of DN Tim cells increases in the CVT of HIV-infected women while the transcriptional pattern remains stable. a Mean

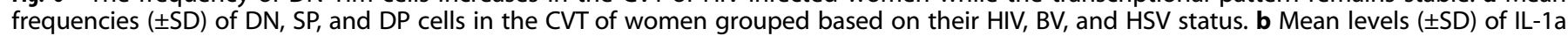

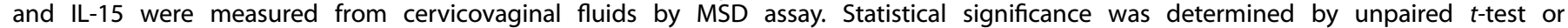

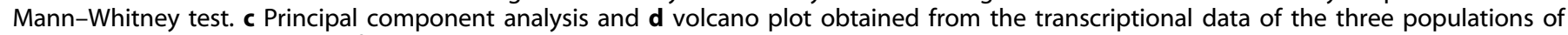
CD8+ T-cells (DN, SP, and DP) from HIV-positive and HIV-negative women

sites, and to further define their functional roles in immune control of pathogens. While it is possible that these cells participate actively in local pathogen control, it is also possible that these cells participate in local inflammation during chronic infection that is detrimental to the host in terms of immunopathology and/or enhancing susceptibility to other local infections. Enhanced knowledge of the specific properties and function of CD8+ Tcells within this tissue site are critical for improving and expediting the design of prevention strategies such as vaccines that target pathogens entering the host across a mucosal barrier tissue.

\section{METHODS}

Study populations

Two Seattle cohorts were used. For the Seattle Surgery Cohort, blood, endocervical, ectocervical and vaginal tissues were 
Table 3. Antibodies list including fluorochromes, clones and distributors

\begin{tabular}{|c|c|c|c|c|}
\hline Reactivity & Target & Fluorochrome & Clone & Company \\
\hline Human & CCR5 & PE CF594 & 2D7/CCR5 & $\mathrm{BD}$ \\
\hline Human & CCR7 & BV510 & $\mathrm{G} 043 \mathrm{H} 7$ & Biolegend \\
\hline Human & CXCR3 & BUV395 & 1C6/CXCR3 & $\mathrm{BD}$ \\
\hline Human & CXCR6 & $\mathrm{PE}$ & K041E5 & Biolegend \\
\hline Human & CX3CR1 & BV421 & 2A9-1 & Biolegend \\
\hline Human & CD3 & BUV496 & UCHT1 & $\mathrm{BD}$ \\
\hline Human & CD4 & APC Cy7 & RPA-T4 & Biolegend \\
\hline Human & CD8 & APC H7 & SK1 & $\mathrm{BD}$ \\
\hline Human & $\mathrm{CD} 25$ & BUV570 & $2 \mathrm{~A} 3$ & $\mathrm{BD}$ \\
\hline Human & CD38 & BUV661 & HIT2 & $\mathrm{BD}$ \\
\hline Human & CD69 & BUV737 & FN50 & $\mathrm{BD}$ \\
\hline Human & CD45 & BUV805 & HI30 & $\mathrm{BD}$ \\
\hline Human & CD45RA & BV570 & HI100 & Biolegend \\
\hline Human & CD49A & BV711 & SR84 & $\mathrm{BD}$ \\
\hline Human & CD101 & AF647 & BB27 & Biolegend \\
\hline Human & CD103 & BV605 & Ber-ACT8 & Biolegend \\
\hline Human & CD127 & BV786 & HIL-7R-M21 & $\mathrm{BD}$ \\
\hline Human & HLA-DR & AF488 & G46-6 & $\mathrm{BD}$ \\
\hline Human & PD-1 & PE Cy7 & EH12.1 & Biolegend \\
\hline Human & GranzymeB & Pac Blue & GB11 & Biolegend \\
\hline Human & Helios & AF647 & $22 \mathrm{~F} 6$ & Biolegend \\
\hline Human & CD4 & APC R700 & RPA-T4 & $\mathrm{BD}$ \\
\hline Human & CCR5 & APC Cy7 & $\mathrm{J} 418 \mathrm{~F} 1$ & Biolegend \\
\hline Human & CD8 & PerCP Cy5.5 & SK1 & $\mathrm{BD}$ \\
\hline Human & IFN-g & Pac Blue & B27 & $\mathrm{BD}$ \\
\hline Human & TNF-a & BV650 & Mab11 & $\mathrm{BD}$ \\
\hline Human & GranzymeB & AF647 & $11 \mathrm{~A} 9$ & $\mathrm{BD}$ \\
\hline Human & IL-17 & APC Cy7 & RPA-T4 & $\mathrm{BD}$ \\
\hline Human & IL-4 & AF488 & 8D4-8 & $\mathrm{BD}$ \\
\hline Human & TGF-b & $\mathrm{PE}$ & N49-653 & $\mathrm{BD}$ \\
\hline Human & IL-2 & PE CF594 & 5344.111 & $\mathrm{BD}$ \\
\hline
\end{tabular}

collected from healthy HIV-negative women undergoing elective hysterectomy. The second cohort included a total of six HIVuninfected and five HIV-infected individuals from the Seattle Vaccine Trials Unit (SVTU). We received biopsy samples for five and four of the donors, respectively. Demographic details are shown in Table 2 and Supplementary Table 1. Our study was approved by the Internal Review Boards at the University of Washington and Fred Hutchinson Cancer Research Center. Informed consent was obtained from all study participants. See additional details in the Supplementary Methods.

Immune phenotyping and intracellular staining

For immune phenotyping, single-cell suspensions from tissues and PBMC were freshly stained with antibodies listed in Table 3. See additional details in the Supplementary Methods.

Cell sorting and RNA sequencing

Single-cell suspensions were stained as previously described and sorted with a FACSAria II (BD, San Jose, CA). Immediately after sorting, RNA was reverse transcribed and amplified following the protocol provided for the SMART-Seq V4 kit. Library preparation and Illumina next generation sequencing were performed at Benaroya Research Institute (Genomic Core) in Seattle. See additional details in the Supplementary Methods. Our data were deposited on Gene Expression Omnibus database (series GSE127834).

\section{ACKNOWLEDGEMENTS}

We wish to thank all the women that have generously donated specimens and time for this study, as well as Noel Williams, Gina Braun, Niall Moren, and all of the staff at the Seattle Vaccine Trial Unit Clinic for their help in enrollment and sample collection for the Seattle HIV Cohort. We thank Vivian Gersuk and the BRI Genomic Core personnel for their assistance with RNA sequencing, and Eric Sandberg from MSD Technology for his help with cytokine quantitation. Finally, we thank the members of the Lund and Prlic labs for helpful discussions. This study was funded by the following grants: R21 Al125025 to L.P., R01 Al121129 to J.M.L. and M.P., and R01 Al131914 to J.M.L.

\section{AUTHOR CONTRIBUTIONS}

Conceptualization: L.P., J.M.L., F.H., M.P.; Formal analyses: S.P., O.H.; Investigation: L.P., A.W.D., F.M. Contributor roles: S.M.H., G.M.L., A.C.K., M.F.F., F.H.; Funding acquisition: L.P., J.M.L., M.P.; Supervision: J.M.L., M.P.; Writing original draft: L.P.; Writing, reviewing and editing: L.P., J.M.L.

\section{ADDITIONAL INFORMATION}

The online version of this article (https://doi.org/10.1038/s41385-019-0186-9) contains supplementary material, which is available to authorized users.

Competing interests: The authors declare no competing interests.

Publisher's note: Springer Nature remains neutral with regard to jurisdictional claims in published maps and institutional affiliations.

\section{REFERENCES}

1. Klenerman, P. \& Hill, A. T cells and viral persistence: lessons from diverse infections. Nat. Immunol. 6, 873-879 (2005).

2. Prlic, M., Williams, M. A. \& Bevan, M. J. Requirements for CD8 T-cell priming, memory generation and maintenance. Curr. Opin. Immunol. 19, 315-319 (2007).

3. Zhang, L. \& Romero, P. Metabolic control of CD8(+) T cell fate decisions and antitumor immunity. Trends Mol. Med. 24, 30-48 (2018).

4. Sallusto, F., Lenig, D., Forster, R., Lipp, M. \& Lanzavecchia, A. Two subsets of memory $\mathrm{T}$ lymphocytes with distinct homing potentials and effector functions. Nature 401, 708-712 (1999).

5. Gebhardt, T. et al. Memory T cells in nonlymphoid tissue that provide enhanced local immunity during infection with herpes simplex virus. Nat. Immunol. 10, 524-530 (2009).

6. Mackay, L. K. et al. Long-lived epithelial immunity by tissue-resident memory T (TRM) cells in the absence of persisting local antigen presentation. Proc. Natl Acad. Sci. USA 109, 7037-7042 (2012).

7. Sathaliyawala, T. et al. Distribution and compartmentalization of human circulating and tissue-resident memory T cell subsets. Immunity 38, 187-197 (2013).

8. Schenkel, J. M., Fraser, K. A., Vezys, V. \& Masopust, D. Sensing and alarm function of resident memory CD8(+) T cells. Nat. Immunol. 14, 509-513 (2013).

9. Schenkel, J. M. \& Masopust, D. Tissue-resident memory T cells. Immunity 41, 886-897 (2014).

10. Shin, H. \& Iwasaki, A. Tissue-resident memory T cells. Immunol. Rev. 255, 165-181 (2013).

11. Wakim, L. M., Woodward-Davis, A. \& Bevan, M. J. Memory T cells persisting within the brain after local infection show functional adaptations to their tissue of residence. Proc. Natl Acad. Sci. USA 107, 17872-17879 (2010).

12. Milner, J. J. \& Goldrath, A. W. Transcriptional programming of tissue-resident memory CD8(+) T cells. Curr. Opin. Immunol. 51, 162-169 (2018).

13. Milner, J. J. et al. Runx3 programs CD8(+) T cell residency in non-lymphoid tissues and tumours. Nature 552, 253-257 (2017).

14. Mackay, L. K. et al. Cutting edge: CD69 interference with sphingosine-1phosphate receptor function regulates peripheral T cell retention. J. Immunol. 194, 2059-2063 (2015).

15. Skon, C. N. et al. Transcriptional downregulation of S1pr1 is required for the establishment of resident memory CD8+T cells. Nat. Immunol. 14, 1285-1293 (2013).

16. Casey, K. A. et al. Antigen-independent differentiation and maintenance of effector-like resident memory $T$ cells in tissues. J. Immunol. 188, 4866-4875 (2012). 
17. Kohlmeier, J. E., Cookenham, T., Roberts, A. D., Miller, S. C. \& Woodland, D. L. Type I interferons regulate cytolytic activity of memory CD8(+) T cells in the lung airways during respiratory virus challenge. Immunity 33, 96-105 (2010).

18. Lopez-Cabrera, M. et al. Transcriptional regulation of the gene encoding the human C-type lectin leukocyte receptor AIM/CD69 and functional characterization of its tumor necrosis factor-alpha-responsive elements. J. Biol. Chem. 270, 21545-21551 (1995).

19. Zhang, N. \& Bevan, M. J. Transforming growth factor-beta signaling controls the formation and maintenance of gut-resident memory $\mathrm{T}$ cells by regulating migration and retention. Immunity 39, 687-696 (2013).

20. Buggert, $M$. et al. Identification and characterization of HIV-specific resident memory CD8(+) T cells in human lymphoid tissue. Sci. Immunol. 3, https://doi. org/10.1126/sciimmunol.aar4526 (2018).

21. Kumar, B. V. et al. Human tissue-resident memory $T$ cells are defined by core transcriptional and functional signatures in lymphoid and mucosal sites. Cell Rep. 20, 2921-2934 (2017)

22. Pallett, L. J. et al. IL-2high tissue-resident T cells in the human liver: sentinels for hepatotropic infection. J. Exp. Med. 214, 1567-1580 (2017).

23. Turner, D. L. et al. Lung niches for the generation and maintenance of tissueresident memory T cells. Mucosal Immunol. 7, 501-510 (2014).

24. Cheuk, S. et al. CD49a expression defines tissue-resident CD8+T cells poised for cytotoxic function in human skin. Immunity 46, 287-300 (2017).

25. Freeman, E. E. et al. Herpes simplex virus 2 infection increases HIV acquisition in men and women: systematic review and meta-analysis of longitudinal studies. AIDS 20, 73-83 (2006).

26. Looker, K. J. et al. Effect of HSV-2 infection on subsequent HIV acquisition: an updated systematic review and meta-analysis. Lancet Infect. Dis. 17, 1303-1316 (2017).

27. Wald, A. \& Link, K. Risk of human immunodeficiency virus infection in herpes simplex virus type 2-seropositive persons: a meta-analysis. J. Infect. Dis. 185, 45-52 (2002)

28. Joag, V. et al. Impact of standard bacterial vaginosis treatment on the genital microbiota, immune milieu, and ex vivo human immunodeficiency virus susceptibility. Clin. Infect. Dis., https://doi.org/10.1093/cid/ciy762 (2018).

29. Rebbapragada, A. et al. Negative mucosal synergy between Herpes simplex type 2 and HIV in the female genital tract. AIDS 21, 589-598 (2007).

30. Gumbi, P. P. et al. Impact of mucosal inflammation on cervical human immunodeficiency virus (HIV-1)-specific CD8 T-cell responses in the female genital tract during chronic HIV infection. J. Virol. 82, 8529-8536 (2008).

31. Roberts, L. et al. Genital tract inflammation during early HIV-1 infection predicts higher plasma viral load set point in women. J. Infect. Dis. 205, 194-203 (2012).

32. Shacklett, B. L., Critchfield, J. W., Ferre, A. L. \& Hayes, T. L. Mucosal T-cell responses to HIV: responding at the front lines. J. Intern. Med. 265, 58-66 (2009).

33. Lee, Y. T. et al. Environmental and antigen receptor-derived signals support sustained surveillance of the lungs by pathogen-specific cytotoxic T lymphocytes. J. Virol. 85, 4085-4094 (2011).

34. Masopust, D., Vezys, V., Wherry, E. J., Barber, D. L. \& Ahmed, R. Cutting edge: gut microenvironment promotes differentiation of a unique memory CD8 T cell population. J. Immunol. 176, 2079-2083 (2006).

35. Watanabe, R. et al. Human skin is protected by four functionally and phenotypically discrete populations of resident and recirculating memory $T$ cells. Sci. Transl. Med. 7, 279ra239 (2015).

36. Gibbons, D. L. et al. Cutting Edge: regulator of G protein signaling-1 selectively regulates gut $\mathrm{T}$ cell trafficking and colitic potential. J. Immunol. 187, 2067-2071 (2011).
37. Fernandez, I. et al. CD101 surface expression discriminates potency among murine FoxP3+regulatory T cells. J. Immunol. 179, 2808-2814 (2007).

38. Herndler-Brandstetter, D. et al. KLRG1(+) effector CD8(+) T cells lose KLRG1, differentiate into all memory $t$ cell lineages, and convey enhanced protective immunity. Immunity 48, 716-729 e718 (2018).

39. Bull, M. E. et al. HIV-1 shedding from the female genital tract is associated with increased Th1 cytokines/chemokines that maintain tissue homeostasis and proportions of CD8+FOXP3+T cells. J. Acquir. Immune Defic. Syndr. 67, 357-364 (2014).

40. Herold, B. C. et al. Plasma and mucosal HIV viral loads are associated with genital tract inflammation in HIV-infected women. J. Acquir. Immune Defic. Syndr. 63, 485-493 (2013).

41. Keller, M. J. et al. Longitudinal assessment of systemic and genital tract inflammatory markers and endogenous genital tract e. coli inhibitory activity in HIVinfected and uninfected women. Am. J. Reprod. Immunol. 75, 631-642 (2016).

42. Mauck, C. et al. Biomarkers of cervical inflammation and immunity associated with cervical shedding of HIV-1. AIDS Res. Hum. Retrovir. 32, 443-451 (2016).

43. Alcaide, M. L. et al. High levels of inflammatory cytokines in the reproductive tract of women with BV and engaging in intravaginal douching: a cross-sectional study of participants in the women interagency HIV study. AIDS Res. Hum. Retrovir. 33, 309-317 (2017).

44. Mitchell, C. M. et al. Bacterial vaginosis, not HIV, is primarily responsible for increased vaginal concentrations of proinflammatory cytokines. AIDS Res. Hum. Retrovir. 24, 667-671 (2008). https://doi.org/10.1089/aid.2008.026810.1089/ aid.2007.0268.

45. Shannon, B. et al. Distinct effects of the cervicovaginal microbiota and herpes simplex type 2 infection on female genital tract immunology. J. Infect. Dis. 215, 1366-1375 (2017).

46. Mogensen, T. H., Melchjorsen, J., Malmgaard, L., Casola, A. \& Paludan, S. R. Suppression of proinflammatory cytokine expression by herpes simplex virus type 1 . J. Virol. 78, 5883-5890 (2004).

47. Clark, R. A. et al. Skin effector memory $T$ cells do not recirculate and provide immune protection in alemtuzumab-treated CTCL patients. Sci. Transl. Med. 4 117 ra117 (2012).

48. Clark, R. A. Resident memory T cells in human health and disease. Sci. Transl. Med. 7, $269 r v 261$ (2015).

49. Willars, G. B. Mammalian RGS proteins: multifunctional regulators of cellular signalling. Semin. Cell Dev. Biol. 17, 363-376 (2006).

50. Moratz, C., Harrison, K. \& Kehrl, J. H. Role of RGS proteins in regulating the migration of B lymphocytes. Arch. Immunol. Ther. Exp. 52, 27-35 (2004).

51. Agenes, F., Bosco, N., Mascarell, L., Fritah, S. \& Ceredig, R. Differential expression of regulator of G-protein signalling transcripts and in vivo migration of CD4+ naive and regulatory T cells. Immunology 115, 179-188 (2005).

52. Brandt, S. M., Mariani, R., Holland, A. U., Hope, T. J. \& Landau, N. R. Association of chemokine-mediated block to HIV entry with coreceptor internalization. J. Biol. Chem. 277, 17291-17299 (2002).

53. Gibbs, A. et al. Human immunodeficiency virus-infected women have high numbers of CD103-CD8+T cells residing close to the basal membrane of the ectocervical epithelium. J. Infect. Dis. 218, 453-465 (2018).

54. Moylan, D. C. et al. Diminished CD103 (alphaEbeta7) expression on resident T cells from the female genital tract of HIV-positive women. Pathog. Immun. 1, 371-387 (2016).

55. Bangs, S. C., McMichael, A. J. \& Xu, X. N. Bystander T cell activation-implications for HIV infection and other diseases. Trends Immunol. 27, 518-524 (2006).

56. Kedl, R. M. \& White, J. T. Foreign antigen-independent memory-phenotype CD4 (+) T cells: a new player in innate immunity? Nat. Rev. Immunol. 18, 1 (2018). 